\title{
Controlled trial comparing prednisolone with an elemental diet plus non-absorbable antibiotics in active Crohn's disease
}

\author{
S SAVERYMUTTU, H J F HODGSON, AND V S CHADWICK \\ From the Department of Medicine, Royal Postgraduate Medical School, London
}

SUMMARY In a randomised clinical trial, patients with moderately active Crohn's disease received either prednisolone $0.5 \mathrm{mg} / \mathrm{kg} /$ day plus a normal diet, or an elemental diet plus oral framycetin, colistin and nystatin. Patients were assessed using the Crohn's disease activity index (CDAI), ESR, and faecal granulocyte excretion quantified by ${ }^{111}$ In-autologous leucocytes. Five patients were intolerant of the elemental diet plus antibiotics and were withdrawn from the trial within 72 hours. Sixteen patients completed 10 days treatment on each regime. Fifteen of 16 patients on elemental diet plus antibiotics and all 16 patients on prednisolone improved with marked, but statistically indistinguishable falls in CDAI, ESR, and faecal granulocyte excretion between the two groups. Thus a regime decreasing the intraluminal concentration of bacteria and complex food molecules, was associated with rapid improvement in activity of Crohn's disease. This suggests that these intraluminal factors play a role in maintaining inflammation and that their removal or alteration offers an approach to management.

The cause of Crohn's disease remains obscure, and treatment unsatisfactory. Much evidence suggests that the expression of immune responses in the gastrointestinal mucosa may be of importance, and evidence for sensitisation of patients to a variety of gut associated antigens, both intrinsic and extrinsic, has been reported. ${ }^{1}$

Surgical diversion of the faecal stream, ${ }^{2}$ total parental nutrition, ${ }^{3}$ the use of elemental diets, ${ }^{4}$ and reduction of bacterial colonisation of the gut, ${ }^{5}$ have all been reported to be of possible benefit in the treatment of Crohn's disease. Most of these reports have been of uncontrolled trials, which is clearly unsatisfactory in a disease characterised by spontaneous remissions and relapses; furthermore the means of assessing changes in disease activity have sometimes been highly subjective, without objective laboratory evidence of reduction in inflammation. We report here the results of a randomised trial comparing the conventional therapy for Crohn's disease by oral prednisolone (the most beneficial treatment overall in the multicentre United States ${ }^{6}$ and European trials ${ }^{7}$ ) with a regime designed to remove antigens from the gut lumen - an elemental

Address for correspondence: Dr H J F Hodgson, RPMS, Hammersmith Hospital, Ducane Road, London W12 0HS.

Received for publication 28 May 1985 diet combined with oral administration of nonabsorbable antibiotics. Assessment of disease activity was with a combination of a clinical index, the sedimentation rate, and the objective technique of measurement of faecal granulocyte excretion.

\section{Methods}

PATIENTS

Thirty seven patients with symptoms of active Crohn's disease were admitted to hospital, the nature of the trial fully explained and consent obtained. Patients were randomised to group A $0.5 \mathrm{mg} / \mathrm{kg} /$ day prednisolone, and normal diet, or group B, an elemental diet (Vivonex, 1800-2400 $\mathrm{ml} / \mathrm{day}$ via fine bore nasogastric tube) plus oral framycetin (500 mg qds), colistin ( 1.5 mega U qds) and nystatin (1 mega U qds). Patients previously taking salazopyrine at the time of the development of active symptoms were left on this medication.

Five patients were intolerant of the group $B$ regime - three developing vomiting attributed to the antibiotics, one being intolerant of a nasogastric tube, and one developing severe diarrhoea which remitted immediately the elemental diet was discontinued. These patients were withdrawn from the trial within 72 hours, and restricted randomisation completed so that group A and group B each 
comprised 16 patients. Group A contained six men and 10 women, age range $22-60$ years, and group B contained six men and 10 women, age range 16-66 years. In each group, three patients were experiencing their first episode of active Crohn's disease. The mean time since diagnosis was 4.3 years in group $A$, and four years in group B. The distribution of disease is shown in Table 1. At the time of admission to the trial, seven out of 10 patients in group A with colonic involvement were receiving salazopyrine, and eight out of 10 patients in group $B$.

The Crohn's Disease Activity Index 9 and ESR were measured on admission to the trial and after 10 days treatment. A ${ }^{111}$ In-leucocyte scan was done two days before initiation of either regime A or B, and a faecal collection made for four days in all. This assessment was also repeated after 10 days of each regime. For leucocyte scanning, in brief, autologous leucocytes were isolated from $50 \mathrm{ml}$ of venous blood, labelled in vitro with ${ }^{111}$ Indium (Amersham International) by a tropolonate chelate, and reinjected (total dose $30-300 \mu \mathrm{Ci}$ ) intravenously. ${ }^{8}$ Abdominal radioactivity was imaged using a gamma camera, and faecal radioactivity counted in an ARMAC counter, and expressed as per cent of injected dose.

After the final assessment, at the time that a normal diet was reinstituted and antibiotics discontinued in group B, patients were treated with prednisolone (10 mg daily) and salazopyrine (if colonic involvement was present).

\section{Results}

At admission to the trial active inflammation of the

Table 1a Results of CDAI, ESR and faecal granulocyte excretion before and after 10 days therapy with prednisolone $(0.5 \mathrm{mg} / \mathrm{kg} /$ day $)$ orally

\begin{tabular}{llcc}
\hline & $C D A I$ & ESR & Faecal excretion \\
\hline Ileal & $239 \rightarrow 90$ & $29 \rightarrow 28$ & $18 \cdot 6 \rightarrow 2 \cdot 5$ \\
& $189 \rightarrow 91$ & $20 \rightarrow 17$ & $5 \cdot 8 \rightarrow 1 \cdot 6$ \\
$331 \rightarrow 221$ & $15 \rightarrow 10$ & $27 \rightarrow 9 \cdot 6$ \\
& $202 \rightarrow 187$ & $45 \rightarrow 24$ & $6 \cdot 6 \rightarrow 4 \cdot 7$ \\
& $83 \rightarrow 34$ & $20 \rightarrow 5$ & $2 \cdot 9 \rightarrow 2 \cdot 4$ \\
& $217 \rightarrow 173$ & $48 \rightarrow 17$ & $20 \cdot 8 \rightarrow 11 \cdot 3$ \\
Ileocolonic & $154 \rightarrow 106$ & $12 \rightarrow 9$ & $10 \cdot 2 \rightarrow 2 \cdot 9$ \\
Colonic & $250 \rightarrow 70$ & $128 \rightarrow 17$ & $31 \cdot 1 \rightarrow 1 \cdot 7$ \\
& $234 \rightarrow 160$ & $122 \rightarrow 42$ & $28 \cdot 6 \rightarrow 20 \cdot 8$ \\
& $348 \rightarrow 182$ & $100 \rightarrow 30$ & $52 \rightarrow 29 \cdot 1$ \\
& $249 \rightarrow 156$ & $42 \rightarrow 20$ & $29 \cdot 9 \rightarrow 19 \cdot 1$ \\
& $256 \rightarrow 231$ & $105 \rightarrow 120$ & $47 \cdot 4 \rightarrow 42 \cdot 8$ \\
& $238 \rightarrow 68$ & $104 \rightarrow 37$ & $23 \cdot 3 \rightarrow 5 \cdot 2$ \\
& $170 \rightarrow 96$ & $40 \rightarrow 16$ & $17 \cdot 3 \rightarrow 5 \cdot 1$ \\
& $210 \rightarrow 194$ & $38 \rightarrow 33$ & $27 \cdot 3 \rightarrow 24 \cdot 6$ \\
& $240 \rightarrow 125$ & $55 \rightarrow 52$ & $32 \cdot 1 \rightarrow 9 \cdot 9$ \\
\hline
\end{tabular}

gastrointestinal tract was confirmed by an abnormal ${ }^{111}$ Indium granulocyte scan in all patients (Fig. 1a). Faecal granulocyte excretion was raised in all patients (normal $<2 \%$ ). Table 2 shows the mean Crohn's Disease Activity Index, the ESR and the faecal granulocyte excretion in patients in both treatment groups, before and after therapy, and full details according to disease distribution are given in Table 1.

Fifteen of 16 patients treated with an elemental diet and non-absorbable antibiotics improved with falls in faecal granulocyte excretion while a single patient deteriorated with a rise in granulocyte excretion (Fig. 2 Table 2). There were concordant changes in the clinical index (CDAI) in 14 out of the 16 patients, including the single patient who deteriorated. The discordant CDAI results were due to alterations in the patients' own assessment of their well being, in one case corresponding to an intercurrent chest infection, and in the other to the development of heartburn. Changes in ESR were also concordant with changes in faecal excretion in 14 of 16 patients; in one patient the ESR rose from 60 to $70 \mathrm{~mm}$ in the first hour despite definite improvement on both faecal excretion and CDAI and in one patient the ESR rose from 10 to $11 \mathrm{~m} / \mathrm{h}$. Figure 1b illustrates the reduction in granulocyte accumulation in areas of inflamed bowel after 10 days of this treatment.

Faecal granulocyte excretion and CDAI fell in all 16 patients treated with prednisolone (Fig. 2 Table 2). Erythrocyte sedimentation rate changed concordantly in 15 of the 16 patients, the exception being the patient showing the smallest decrease in faecal granulocyte excretion. The details of the measured

Table 1b Results of CDAI, ESR and faecal granulocyte excretion before and after 10 days therapy with elemental diet plus non-absorbable antibiotics

\begin{tabular}{llcc}
\hline & $C D A I$ & $E S R$ & Faecal excretion \\
\hline Ileal & $197 \rightarrow 78$ & $55 \rightarrow 26$ & $36 \cdot 3 \rightarrow 4 \cdot 7$ \\
& $95 \rightarrow 51$ & $10 \rightarrow 11$ & $9 \cdot 8 \rightarrow 3 \cdot 2$ \\
& $170 \rightarrow 176$ & $11 \rightarrow 2$ & $4 \cdot 3 \rightarrow 2 \cdot 6$ \\
& $310 \rightarrow 338$ & $33 \rightarrow 20$ & $18 \cdot 2 \rightarrow 5 \cdot 2$ \\
& $239 \rightarrow 213$ & $30 \rightarrow 4$ & $17 \cdot 2 \rightarrow 7 \cdot 2$ \\
& $358 \rightarrow 260$ & $60 \rightarrow 70$ & $25 \cdot 5 \rightarrow 12 \cdot 1$ \\
& $250 \rightarrow 172$ & $55 \rightarrow 20$ & $36 \cdot 1 \rightarrow 33 \cdot 6$ \\
Ileocolonic & $177 \rightarrow 50$ & $26 \rightarrow 22$ & $12 \cdot 7 \rightarrow 3 \cdot 2$ \\
& $185 \rightarrow 123$ & $36 \rightarrow 20$ & $28 \cdot 6 \rightarrow 5 \cdot 2$ \\
& $386 \rightarrow 87$ & $100 \rightarrow 88$ & $31 \cdot 3 \rightarrow 4 \cdot 8$ \\
Colonic & $280 \rightarrow 153$ & $90 \rightarrow 45$ & $34 \cdot 8 \rightarrow 4 \cdot 9$ \\
& $221 \rightarrow 75$ & $6 \rightarrow 5$ & $7 \cdot 3 \rightarrow 1 \cdot 1$ \\
& $201 \rightarrow 248$ & $37 \rightarrow 55$ & $16 \cdot 2 \rightarrow 32 \cdot 1$ \\
& $185 \rightarrow 152$ & $70 \rightarrow 18$ & $31 \rightarrow 20 \cdot 1$ \\
& $147 \rightarrow 199$ & $24 \rightarrow 17$ & $25 \cdot 6 \rightarrow 15 \cdot 2$ \\
& $148 \rightarrow 136$ & $45 \rightarrow 9$ & $26 \cdot 1 \rightarrow 3 \cdot 1$ \\
\hline
\end{tabular}




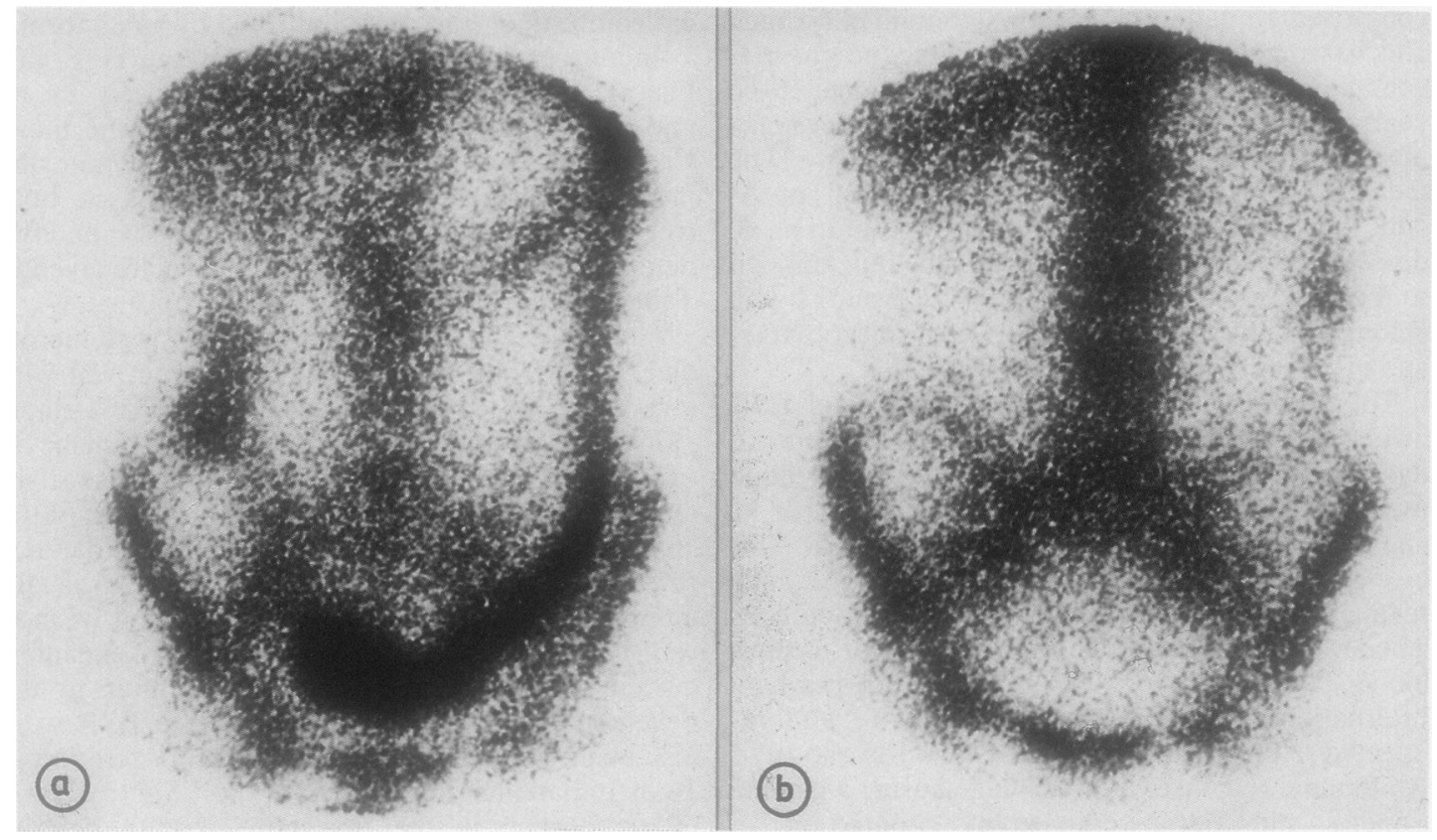

Fig. $1{ }^{111}$ In-WBC scan, four hours after injection of autologous labelled granulocytes; (a) before, (b) after, treatment with an elemental diet plus non-absorbable antibiotics. Labelled neutrophils before treatment accumulate throughout the colon, particularly in descending, pelvic and sigmoid area. After treatment, the cells accumulate over the bone marrow and liver, the pattern seen in the absence of inflammatory foci.

indices in the two groups of patients before and after therapy (Table 2) indicate that there were no differences between the levels of any of these parameters between the two groups at admission to the trial. There was a significant fall in parameters of disease activity after 10 days in both treatment groups $(p<0.01)$. At the end of the trial there was no significant difference in the levels between parameters of disease activity between the two treatment groups.

\section{Discussion}

The course of Crohn's disease is unpredictable, and spontaneous fluctuations in activity are common, indicating the need for controlled evaluation of therapy. Such trials have shown convincing effects of corticosteroids in the treatment of active Crohn's disease both affecting the small intestine and the colon. ${ }^{67}$ The modes of assessment in such trials have been criticised, however, particularly when they have been mainly based on the use of a clinical disease activity index such as the CDAI. This numerical index,${ }^{9}$ derived for the purpose of subsequent statistical analysis, is based on surveying eight clinical features to reach a score ranging between 50 and over $450 ; 23 \%$ of the variance of the final value depends upon the patients subjective assessment of his own well being. The method is thus an imperfect tool in evaluating the therapeutic effects of drugs such as corticosteroids which may have prominent euphoriant effects. While laboratory measurements of acute phase reactants, or the sedimentation rate, do reflect mucosal inflammation, they lack specificity for gastrointestinal disease and are easily affected by intercurrent episodes. The technique of ${ }^{111} \mathrm{In}$ dium scanning, however, with measurement of faecal radioactivity excretion, offers an objective

Table 2 Faecal granulocyte excretion, ESR and CDAI in groups $A$ and $B$ before and after treatment

\begin{tabular}{lcccc}
\hline & $\begin{array}{l}\text { A-Corticosteroid } \\
\text { therapy and normal }\end{array}$ & \multicolumn{2}{l}{$\begin{array}{l}\text { B-Elemental diet plus } \\
\text { antibiotics }\end{array}$} \\
\hline & Pre $R_{X}$ & Post $R_{X}$ & Pre $R_{X}$ & Post $R_{X}$ \\
\hline Faecal granulocyte & & & & \\
excretion \pm SEM & $23 \cdot 9 \pm 4$ & $12 \cdot 1 \pm 3$ & $22 \cdot 5 \pm 3$ & $9 \cdot 9 \pm 2$ \\
ESR mm/h \pm SEM & $58 \pm 9$ & $31 \pm 8$ & $45 \pm 6 \cdot 8$ & $27 \pm 6$ \\
CDAI \pm SEM & $226 \pm 16$ & $130 \pm 15$ & $222 \pm 20$ & $156 \pm 20$ \\
\hline
\end{tabular}




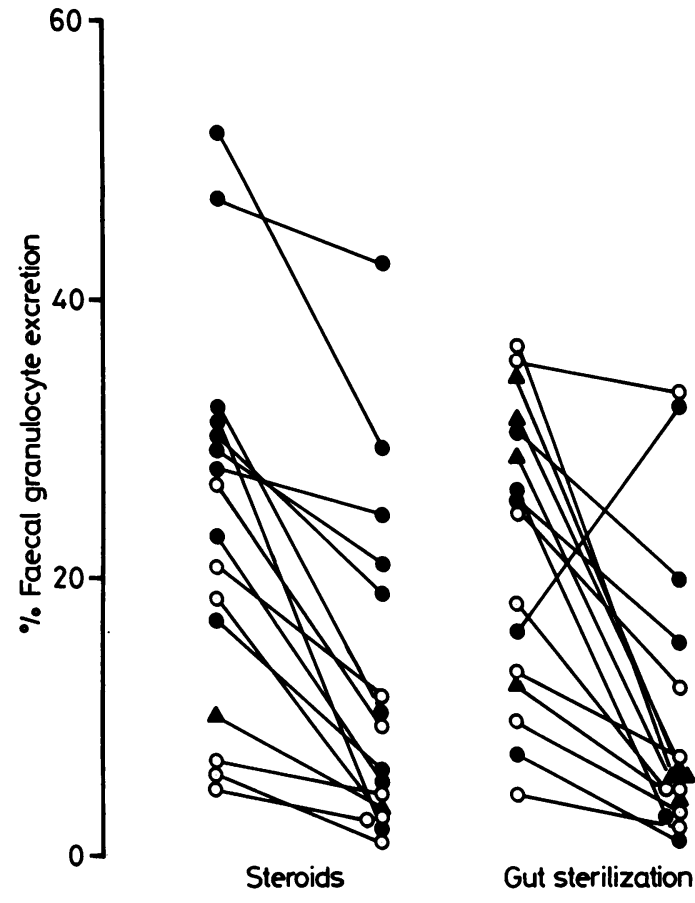

Fig. 2 Faecal granulocyte excretion over four days after injection of ${ }^{111}$ In-labelled WBCs, expressed as \% of total radioactivity injected.

measurement, specific for mucosal inflammation. This study now shows that it is a highly effective means of assessing the effects of drugs in clinical trials, although it is currently technically demanding. As would be expected, some patients with CDAI levels of below 150 , usually taken at clinical remission, in fact had raised faecal granulocyte levels.

In the groups of patients surveyed here, all those treated with corticosteroids rapidly improved by both objective and subjective indices of mucosal inflammation. The striking findings was that in a group of 16 patients treated with oral nonabsorbable antibiotics and an elemental diet, there was a statistically indistinguishable improvement in these parameters of inflammation. The progress was assessed after 10 days therapy, although usually the improvement was clinically apparent within a few days of initiation of treatment. The effect of non-absorbable antibiotics and elemental diet was seen both in patients with colonic and those with small intestinal involvement.

There is evidence from others that an approach involving the removal of antigens from the gastrointestinal tract may be effective. The only con- trolled study, that of O'Morain $e a^{\prime} l^{10}$ showed that an elemental diet - without antibiotics - was as effective after 28 days of therapy as corticosteroids in patients with mild or moderately active disease. Responses to total parental nutrition, ${ }^{3}$ or to nonabsorbable antibiotics, have been reported in other uncontrolled studies. ${ }^{11}$ The combination of the two used here appears to have a particular advantage in that it worked rapidly suggesting that it may be an appropriate form of therapy for ill patients, avoiding the side effects of corticosteroid therapy.

The use of the antibiotic and elemental diet regime was, however, limited - in nearly a quarter of individuals in whom it was tried - by intolerance. This was mainly in the form of vomiting and nausea induced by the antibiotics. A similar high incidence of non-compliance was seen in the controlled trial of elemental diet. ${ }^{10}$ In our study such intolerance became clear within two to three days of initiating treatment, and thus can be recognised early without causing significant delay in treatment.

The effectiveness of this regime of nonabsorbable antibiotics and elemental diet is clearly established by this study, as a short term rapid means of treating acute Crohn's disease. The mechanism of improvement - whether by reducing antigens as targets of the host immune response, by removing the invading bacteria which are reported within the mucosa of patients with Crohn's disease ${ }^{12}$ or by repairing a postulated nutritional defect, remains speculative. The long term outlook of remission induced by this regime also requires to be assessed. In this trial, the reintroduction of a normal diet, and withdrawal of antibiotics, was electively covered with low doses of corticosteroids. Further studies are in process to determine whether this is necessary.

This work was supported by grants from the Wellcome Trust and Amersham International.

\section{References}

1 Hodgson HJF. Immunological aspects of inflammatory bowel disease. In: Brooke BV, Wilkinson A, eds. Inflammatory diseases of the bowel. London: Pitman Medical, 1980: 38-52.

2 Burman JH, Thompson H, Cooke WT, Williams JA. The effects of diversion of intestinal contents on the progress of Crohn's disease of the large bowel. Gut 1979; 12: 11-15.

3 Elson CO, Layden TJ, Nemchausky BA, Rosenberg JC, Rosenberg I. An evaluation of total parenteral nutrition in the management of inflammatory bowel disease. Dig Dis Sci 1980; 25: 42-8.

4 O'Morain C, Segal AW, Levi AJ. Elemental diet as 
primary treatment of acute Crohn's disease: a controlled trial. Br Med J 1980; 281: 1173-5.

5 Moss AA, Carbone JV, Kressel HY. Successful treatment of Crohn's disease with broad spectrum antibiotics: initial and long term results. Gastroenterology 1977; 77: 83.

6 Summers RW, Switz DM, Sessions JT et al. National Cooperative Crohn's disease study: results of drug treatment. Gastroenterology 1979; 77: 847-69.

7 Malchow H, Ewe K, Brandes JW, et al. European Cooperative Crohn's disease study (ECCDS): results of treatment. Gastroenterology 1984; 86: 249-66.

8 Saverymuttu SH, Peters AM, Lavender JP, Pepys MB, Hodgson HJF, Chadwick VS. Quantitative faecal
Indium-111-labelled leucocyte excretion in the assessment of disease activity in Crohn's disease. Gastroenterology 1983; 85: 1333-9.

9 Best WR, Becktel JM, Singleton JW, Keen F. Devellopment of Crohn's disease activity index. Gastroenterology 1976; 70: 785-7.

10 O'Morain C, Segal AW, Levi AJ. Elemental diet as primary treatment of acute Crohn's disease: a controlled trial. $\mathrm{Br}$ Med J 1984; 288: 1859-62.

11 Neale G. The management of Crohn's disease. In: Peters DK, ed. Advanced medicine 12. Tunbridge Wells: Pitmans Medical, 1976: 298-311.

12 Aluwihare APR. Elective microscopy in Crohn's disease. Gut 1971; 12: 509-18. 